\title{
Correction to: Local drivers of the structure of a tropical bird-seed dispersal network
}

\author{
Tiago Machado-de-Souza ${ }^{1,2,3,4}$ [C $\cdot$ Ricardo Pamplona Campos ${ }^{1} \cdot$ Mariano Devoto $^{5,6} \cdot$ Isabela Galarda Varassin $^{1,2}$
}

Published online: 29 April 2019

(c) Springer-Verlag GmbH Germany, part of Springer Nature 2019

\section{Correction to: Oecologia (2019) 189:421-433 \\ https://doi.org/10.1007/s00442-018-4322-0}

Unfortunately, the detailed description of the ESM material went wrong in the published version of the dataset. The correct version is updated here.

The original article can be found online at https://doi.org/10.1007/ s00442-018-4322-0.

Electronic supplementary material The online version of this article (https://doi.org/10.1007/s00442-019-04392-2) contains supplementary material, which is available to authorized users.

Tiago Machado-de-Souza

tigomachado@gmail.com

1 Laboratório de Interações e Biologia Reprodutiva,

Departamento de Botânica, Universidade Federal do Paraná,

Centro Politécnico, Jardim das Américas, Curitiba,

PR 81531-990, Brazil

2 Programa de Pós-Graduação em Ecologia e Conservação, Universidade Federal do Paraná, Centro Politécnico, Jardim das Américas, Curitiba, PR 81531-990, Brazil

3 Mater Natura-Instituto de Estudos Ambientais, Rua Lamenha Lins 1080, Curitiba, PR 80250-020, Brazil

4 Instituto de Estudos Ambientais do Paraná (IEAP), Rua Rômulo Gutierrez 731, Curitiba, PR 80820-260, Brazil

5 Facultad de Agronomía, Cátedra de Botánica General, Universidad de Buenos Aires, Avda. San Martín 4453, C1417DSE Buenos Aires, Argentina

6 Consejo Nacional de Investigaciones Científicas y Técnicas (CONICET), Buenos Aires, Argentina 